\title{
Erenumab in highly therapy-refractory migraine patients: First German real-world evidence
}

Armin Scheffler ${ }^{1}$, Olga Messel ${ }^{1}$, Sebastian Wurthmann ${ }^{1}$, Michael Nsaka ${ }^{1}$, Christoph Kleinschnitz ${ }^{1}$, Martin Glas², Steffen Naegel ${ }^{1,3}$ and Dagny Holle ${ }^{1^{*}}$ (D)

\begin{abstract}
Background: Calcitonin gene related peptide (CGRP) monoclonal antibodies (mAB) are the first specific migraine prophylactic medication. Erenumab is the only CGRP mAB targeting the CGRP receptor. Clinical data regarding efficacy and tolerability of erenumab in highly therapy-refractory patients are not available, yet, although many patients treated with CGRP mAB under real world conditions can be considered as highly therapy-refractory.

Methods: Clinical routine data of highly therapy-refractory migraine patients treated with erenumab $70 \mathrm{mg}$ for 3 months between November 2018 and December 2019 in the West German Headache Center, University Hospital Essen, Germany, were analysed. Monthly migraine days (MMD), monthly headache days (MHD) and days of acute medication intake (AMD) were assessed. Statistical analysis was performed using the Wilcoxon test. Descriptive statistics were performed to evaluate changes of vegetative symptoms, acute medication response, side effects, as well as treatment satisfaction.

Results: Complete clinical data were available for 26 episodic (EM) and 74 chronic (CM) migraineurs. Sixty-six \% $(n=49)$ of $\mathrm{CM}$ patients had an additional medication overuse headache $(\mathrm{MOH})$. After 3 months $57.7 \%$ of EM patients and $41.9 \%$ of CM patients had a $50 \%$ or greater reduction of MMD. The mean number of MMD was reduced by 3.43 (SE 1.26) in EM, and by 4.72 (SE 0.87) in CM. Thirty-nine patients (52.7\%) returned from chronic to episodic course of migraine. After 3 months, 23 patients (46.9\%) were not suffering from a MOH anymore.
\end{abstract}

Conclusions: Erenumab seems to be a promising therapeutic option in highly therapy-refractory migraine patients. Trial registration: Retrospective registered.

Keywords: 1. migraine, 2. erenumab, 3. therapy, 4. real-world, 5. CGRP antibody

\section{Background}

Migraine is the 3rd most prevalent illness in the world and one of the main causes of disability [1]. So far available non-specific prophylactic drug medication was often poorly tolerated and not effective in every patient.

\footnotetext{
* Correspondence: dagny.holle-lee@uk-essen.de

'Department of Neurology, West German Headache Center, University Hospital Essen, University Duisburg-Essen, Hufelandstr. 55, 45147 Essen, Germany

Full list of author information is available at the end of the article
}

Recently, monoclonal CGRP (calcitonin gene related peptide) antibodies (mAB) have become the first specific migraine prophylaxis, which has shown its efficacy in large phase III studies in the treatment of episodic (EM) and chronic migraine $(\mathrm{CM})$ with or without medication overuse headache $(\mathrm{MOH})$ [2-4] and is now available worldwide for migraine treatment.

In Europe, three CGRP mAB, erenumab (Aimovig, Novartis, Basel, Switzerland), fremanezumab (Ajovy ${ }^{\circ}$, Teva, Petach Tikva, Israel) and galcanezumab (Emgality ${ }^{\circ}$,

C C The Author(s). 2020 Open Access This article is licensed under a Creative Commons Attribution 4.0 International License, which permits use, sharing, adaptation, distribution and reproduction in any medium or format, as long as you give appropriate credit to the original author(s) and the source, provide a link to the Creative Commons licence, and indicate if changes were made. The images or other third party material in this article are included in the article's Creative Commons licence, unless indicated otherwise in a credit line to the material. If material is not included in the article's Creative Commons licence and your intended use is not permitted by statutory regulation or exceeds the permitted use, you will need to obtain permission directly from the copyright holder. To view a copy of this licence, visit http://creativecommons.org/licenses/by/4.0/ The Creative Commons Public Domain Dedication waiver (http://creativecommons.org/publicdomain/zero/1.0/) applies to the data made available in this article, unless otherwise stated in a credit line to the data. 
Lilly, Indianapolis, USA) are approved for migraine prevention in patients suffering from at least four migraine days per month and recommended for migraine prevention by current European guidelines [5]. Erenumab is the only substance targeting the CGRP receptor instead of the molecule itself. Thus far, no clear differences in efficacy and tolerability between the individual mABs could be identified.

Clinical trials were only performed in migraine patients who had failed up to four prophylactic medications in the past [2-4]. Although there are indications of good effectiveness under real-world conditions $[6,7]$, data on highly therapy-refractory patients that had failed more than four prophylactic treatments are missing, yet.

We present now clinical data on this highly therapyrefractory migraine population treated with erenumab under real world conditions.

\section{Methods}

We retrospectively analysed routine clinical data of EM and CM patients presented at the West German Headache Center, Department of Neurology, University Hospital Essen, Germany between November 2018 and December 2019. The analysis was approved by the independent ethics committee of the University Hospital Essen. Patients meeting the following criteria were included in the analysis: a) EM/CM patients with at least 4 migraine days a month according to the current diagnostic criteria of the International headache classification (ICHD-3 [8]), b) documented history of the last 90 days prior starting erenumab therapy regarding monthly migraine days (MMD), monthly headache days (MHD) and monthly intake of acute medication (AMD), c) completion of a 90 days treatment interval with monthly 70 mg erenumab d) available complete clinical data including headache diaries and side effects. A paper-based headache diary was used. MMD, MHD and AMD are the average monthly mean values over the respective total observation period of 90 days. A headache day was defined as a day with any kind of headache, a migraine day was defined by patients when they had severe pain, migraine pain characteristics (pulsating, one-sided pain), aura symptoms, vegetative symptoms like phono- or photophobia, nausea, vomiting, need for rest, or when triptans were taken. Most patients answered questionnaires regarding different aspects of migraine: intensity of migraine $(n=95)$, duration of the migraine attack $(n=90)$, effect of acute therapy $(n=90)$, effect on the aura $(n=90)$, need for rest $(n=93)$, dizziness $(n=92)$, nausea $(n=93)$, phono- and photophobia $(n=93)$ as well as therapy satisfaction $(n=93)$. Due to reasons of reimbursement by the German statutory health insurance, all treated patients had tried at least 5 (when EM) and 6 (when $\mathrm{CM}$ ) approved prophylactic drugs previously without sufficient treatment effects, had discontinued those due to side effects, or were not eligible for intake due to contraindication. Approved drug classes were the following: betablockers (metoprolol or propranolol), tricyclic antidepressants (amitriptyline), calcium channel blockers (flunarizine), anticonvulsants (topiramate and valproic acid) and for $\mathrm{CM}$ additionally onabotulinumtoxin A. The pre-existing medication taken for other indications or migraine prophylaxis were not changed and on a stable dosage at least 6 weeks prior to treatment start. No other medication with a potential disease modifying effect was started during the observational period. Data were analysed using SPSS software (IBM SPSS Statistics for Windows, Version 25.0. Armonk, NY: IBM Corp). Wilcoxon's test was used to compare MMD, MHD, and AMD before and after treatment. The test procedure was two-sided, Bonferoni's method for multiple comparisons was set at $p<0.05 / 3=0.016$. Patient reported outcomes were analysed descriptively.

\section{Results}

Clinical data of a total of 100 migraine patients were analysed. Details regarding demographic data and numbers of MMD, MHD, and AMD before and after treatment are stated in Table 1. In brief, most of the patients were female $(82 \% ; n=82), 26 \%(n=26)$ suffered from EM, $74 \%(n=74)$ from CM. Sixty-six $\%(n=49)$ of CM patients had an additional $\mathrm{MOH}$.

After 3 months of erenumab therapy $57.7 \%$ of EM patients $(n=15)$ and $41.9 \%$ of CM patients $(n=31)$ had at least a $50 \%$ reduction in MMD (Fig. 1). Fifty-three \% of $\mathrm{CM}$ patients $(n=39)$ returned from chronic to episodic course of migraine. After 3 months, $46.9 \%$ of $\mathrm{MOH}$ patients $(n=23)$ were not suffering from $\mathrm{MOH}$ anymore.

Subjective improvement of migraine intensity and duration was reported by considerably more than half of the patients ( $n=67,70.5 \%$ and $n=53,58.9 \%$, respectively). Subjective treatment effects on migraine associated vegetative symptoms were rather small. Efficacy of acute medication was largely unchanged under therapy (Fig. 2).

Self-reported general satisfaction with therapy was high (very satisfactory/satisfactory $61,3 \%(n=57)$; moderately satisfactory $20,4 \% \quad(n=19)$; unsatisfactory/very unsatisfactory $18,3 \%(n=17))$.

Out of 100 patients $42 \%(n=42)$ reported side effects. These 42 patients mainly complained digestive problems or constipation $(23,8 \%, n=10)$, injection side skin symptoms or itching $(23,8 \% ; n=10)$, fatigue or a feeling of exhaustion $(16,7 \%, n=7)$, and insomnia $(9,5 \%, n=4)$. One patient discontinued treatment due to severe constipation. 
Table 1 Patients' characteristics and treatment response

\begin{tabular}{|c|c|c|c|c|c|}
\hline \multicolumn{6}{|l|}{ A) Episodic migraine $(n=26)$} \\
\hline \multirow[t]{2}{*}{ Age, y } & \multicolumn{2}{|l|}{$52.9(S D$ 9.1) } & \multicolumn{2}{|c|}{ Sex, female : male } & $23: 3$ \\
\hline & Before treatment, $d$ & After 3 month, $d$ & $P$-value & Change from baseline, $d$ & $50 \%$ response, $\%$ \\
\hline MMD (SD) & $9.42(2.91)$ & $5.99(5.43)$ & $<0.001$ & -3.43 (SE 1.26) & 57.7 \\
\hline $\mathrm{MHD}(\mathrm{SD})$ & $10.40(2.47)$ & $7.06(5.46)$ & 0.001 & -3.33 (SE 1.17) & 46.2 \\
\hline AMD (SD) & $10.22(4.69)$ & $5.74(5.07)$ & $<0.001$ & -4.48 (SE 1.27) & 61.5 \\
\hline \multicolumn{6}{|l|}{ B) Chronic migraine $(n=74)$} \\
\hline Age, y & 45.8 (SD 12.9) & & \multicolumn{2}{|c|}{ Sex, female : male } & $59: 15$ \\
\hline \multirow[t]{2}{*}{$\mathrm{MOH}$ (before treatment), $\mathrm{n}$} & 49 & & $\mathrm{MOH}(\mathrm{aft}$ & month), $\mathrm{n}$ & 26 \\
\hline & Before treatment, $d$ & After 3 month, d & $P$-value & Change from baseline, $d$ & $50 \%$ response, $\%$ \\
\hline MMD (SD) & $15.69(6.64)$ & $10.97(7.81)$ & $<0.001$ & $-4.72($ SE 0.87) & 41.9 \\
\hline $\mathrm{MHD}(\mathrm{SD})$ & $21.27(5.5)$ & $15.8(8.9)$ & $<0.001$ & -5.47 (SE 0.86) & 32.4 \\
\hline $\mathrm{AMD}(\mathrm{SD})$ & $11.62(6.14)$ & $8.86(5.97)$ & $<0.001$ & -2.76 (SE 0.83) & 27.0 \\
\hline
\end{tabular}

Data before treatment reflect the 3 months before start of erenumab treatment. (MMD monthly migraine days, $M H D$ monthly headache days, $A M D$ monthly days of intake of acute migraine medication, $S D$ standard deviation, $S E$ standard error, $y$ years, $d$ days)

\section{Discussion}

Our clinical data show efficacy and tolerability of CGRP $\mathrm{mAB}$ under real world conditions in a German tertiary headache center. Data on this highly therapy-refractory patient population were not available, yet. The results of our analysis look very promising, especially considering the fact that no other approved drug therapies have been available for this patient population.

Efficacy of erenumab in highly therapy-refractory patients is comparable to results from clinical trials of patients that had failed fewer prophylactic medications prior treatment. Under trial conditions migraine days went down between 1.8 and 3.7 days/month [2, $9,10]$ compared to a reduction of 3.43 days/month in EM under real world conditions; MMD 50\% responder rates were between $30 \%$ and $43.3 \%[2,9,10]$ compared to $57.7 \%$ in this real-world analysis. Results from the phase II erenumab CM trial reported a reduction of 6.6 days/month and a $50 \%$ responder rate of $40 \%$ [11] which is comparable to our real world

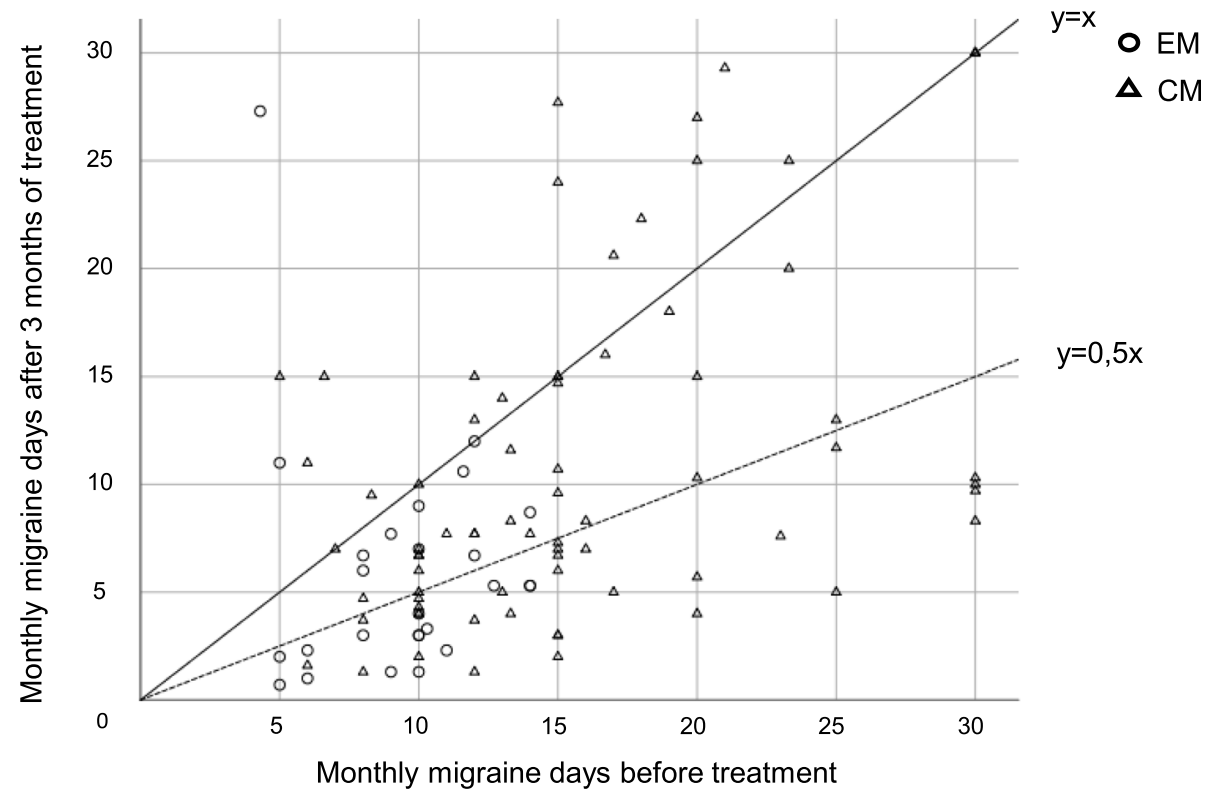

Fig. 1 Therapy response after 3 months of erenumab treatment. Number of monthly migraine days before and after three months of treatment with erenumab. Every plotting symbol represents a patient with EM (circle) or CM (triangle). All symbols on the function $y=0.5 x$ and below show a reduction of at least $50 \%$. The symbols below the function $y=x$ represent patients who still have less monthly migraine days after 3 months of treatment, all symbols on the function or above can be considered as non-responder 


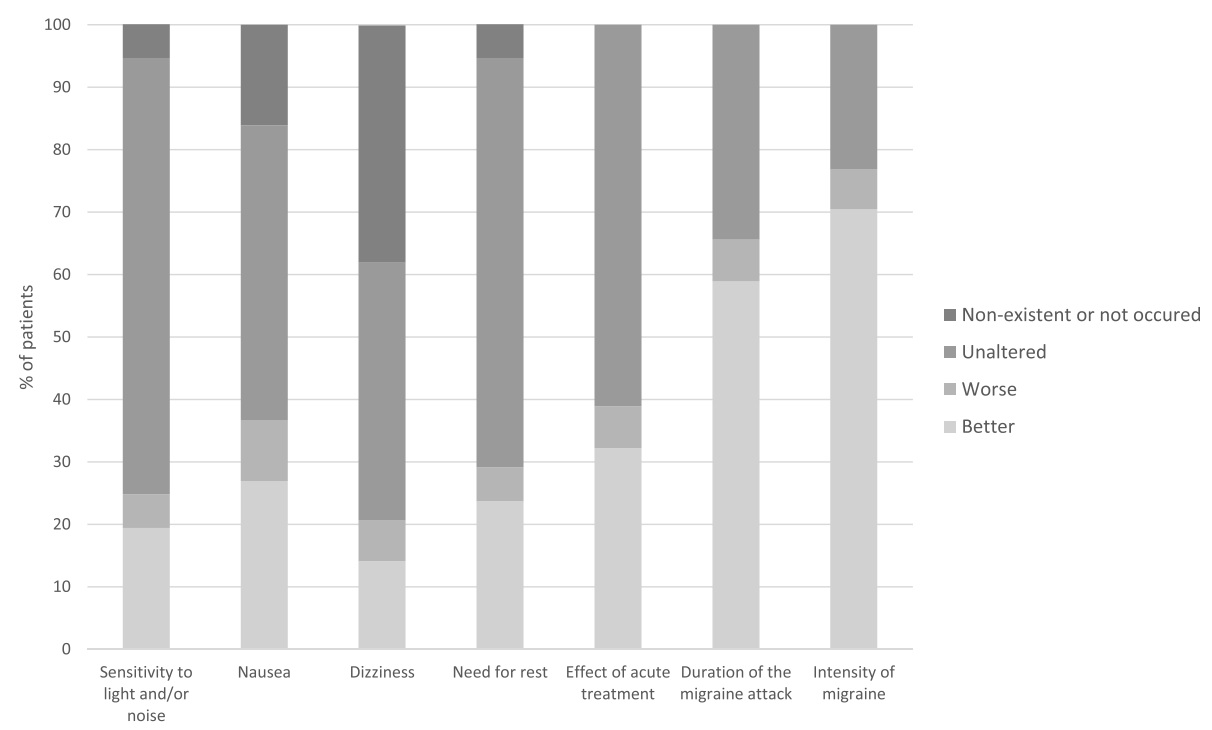

Fig. 2 Distinct treatment responses regarding migraine characteristics. Patient reported subjective treatment response after three months of erenumab therapy. Migraine-associated vegetative symptoms, dizziness, need for rest and effects of acute medications stayed unaffected in the majority of patient, while duration and intensity of migraine improved

data showing a reduction of 4.72 days and a $50 \%$ responder rate of $41.9 \%$. The slightly better treatment effect under real-world conditions compared to previous clinical study results may be explained by extraordinarily high patient expectations regarding the effectiveness of the drug and the lack of a placebo arm. Further data will show to which extent this effect stays stable during longterm treatment.

Compared to the clinical studies, similarly high rates of side effects were found and no serious side effects occurred $[2,9,12]$ supporting a good tolerability of erenumab.

Many patients with highly therapy-refractory migraine suffer from additional $\mathrm{MOH}$, in our real-world population $49 \%$ of all analysed patients. Our data show that erenumab could also be effective in patients with additional $\mathrm{MOH}$ although medication overuse was not stopped prior therapy. Our observations confirm the data of the subgroup analysis of the CM Phase II erenumab study [12], also showing the efficacy of erenumab in $\mathrm{MOH}$. Nevertheless, a migraine day was defined inter alia when migraine specific acute drug intake (triptane) was needed (as described in the method part). Our data can only show a reduction of acute drug intake as well as an improvement of $\mathrm{MOH}$ at the same time. We are not able to distinguish, if erenumab has a direct effect on $\mathrm{MOH}$ or if the reduction of AMD leads to an improvement of the $\mathrm{MOH}$.

Besides reduction of frequency and acute medication intake, the greatest therapeutic effect was seen in reduction of intensity and duration of migraine attacks. The therapeutic effect of erenumab seems to be less pronounced regarding vegetative accompanying symptoms of migraine.
For the majority of our patients, migraine-associated phonophobia, photophobia and nausea remained unchanged. It can only be assumed that vegetative accompanying symptoms are mainly driven by central mechanisms and therefore less influenced by the peripheral acting CGRP antibody whereas migraine pain intensity and frequency might be more peripherally modulated [13].

Shortcoming of this single-center experience are mainly its retrospective nature and that data are mainly based on purely subjective reports of patients. Nevertheless, these data are important from our point of view, as better evaluations regarding headache of this predominantly treated patient collective are still missing. Additionally, patients in our patient population received the lower erenumab dosage of $70 \mathrm{mg}$ instead of the now available $140 \mathrm{mg}$ dosage. There is some evidence that difficult-to-treat patients who failed prophylactic medication in the past may benefit more from $140 \mathrm{mg}$ compared to $70 \mathrm{mg}$ erenumab [14]. However, there are no data of direct comparison between 70 and $140 \mathrm{mg}$ dosage. Whether patients could benefit more from a higher dosage cannot be answered at present.

\section{Conclusion}

Our data show that erenumab is a promising therapy option in highly therapy-refractory migraine patients with or without $\mathrm{MOH}$. Real-world efficacy and tolerability of erenumab seems to be comparable with results from clinical trials, even in more therapy-refractory patients than those treated in clinical studies. Future applications will show whether our results can be confirmed for larger numbers of patients and for CGRP $\mathrm{mAB}$ in general. 


\section{Abbreviations}

AMD: Monthly intake of acute medication; CGRP: Calcitonin gene related peptide; CM: Chronic migraine; EM: Episodic migraine; ICHD-3: International headache classification; mAB: Monoclonal antibody; MHD: Monthly headache days; MMD: Monthly migraine days; MOH: Medication overuse headache; SE: Standard error

\section{Acknowledgements}

Benjamin Kubo corrected the manuscript regarding language. The study is part of the doctor thesis of one of the authors (OM).

\section{Authors' contributions}

AS has the major role in the acquisition of data, analysed and interpreted the data and drafted the manuscript for intellectual content. OM analysed and interpreted the data and revised the manuscript for intellectual content. SW, MN, CK, SN and MG interpreted the data and revised the manuscript for intellectual content. DH designed and conceptualized study, interpreted the data and drafted the manuscript for intellectual content. All authors read and approved the final manuscript.

\section{Funding}

No funding.

\section{Availability of data and materials}

The datasets used and/or analysed during the current study are available from the corresponding author on reasonable request.

\section{Ethics approval and consent to participate}

The analysis was approved by the independent ethics committee of the University Hospital Essen (19-9004-BO) and all patients gave written consent to general analysis of their personal and clinical data.

\section{Consent for publication}

Not applicable.

\section{Competing interests}

AS received travel fees from Teva, honoraria for participation on an advisory board of Novartis. SW reports personal fees from Allergan, personal fees from Tea, personal fees from Novartis, outside the submitted work. MG received honoraria from Novartis, UCB, Teva, Bayer, Novocure, Medac, Merck, Kyowa Kirin, has a consulting or advisory role to declare for Roche, Novartis, AbbVie, Novocure, and Daiichi Synkyo, and received travel fees from Novocure and Medac. SN reports grants from Novartis, personal fees from Novartis, personal fees from Teva, outside the submitted work. DH has received scientific support and/or honoraria from Biogen, Novartis, Lilly, SanofiAventis, Teva, Allergan, Hormosan. OM, MN and CK declare that they have no competing interests.

\section{Author details}

'Department of Neurology, West German Headache Center, University Hospital Essen, University Duisburg-Essen, Hufelandstr. 55, 45147 Essen, Germany. ${ }^{2}$ Department of Neurology, Division of Clinical Neurooncology, University Hospital Essen, University Duisburg-Essen, Hufelandstr. 55, 45147 Essen, Germany. ${ }^{3}$ Department of Neurology, Martin Luther University Halle-Wittenberg, University Hospital Halle, Ernst-Grube-Str. 40, 06097 Halle (Saale), Germany.

Received: 26 March 2020 Accepted: 18 June 2020

Published online: 03 July 2020

\section{References}

1. GBD 2016 Neurology Collaborators (2019) Global, regional, and national burden of neurological disorders, 1990-2016: a systematic analysis for the Global Burden of Disease Study 2016. Lancet Neurol 18:459-480

2. Reuter U, Goadsby PJ, Lanteri-Minet M et al (2018) Efficacy and tolerability of erenumab in patients with episodic migraine in whom two-to-four previous preventive treatments were unsuccessful: a randomised, doubleblind, placebo-controlled, phase 3b study. Lancet Lond Engl 392:2280-2287

3. Ferrari MD, Diener HC, Ning X et al (2019) Fremanezumab versus placebo for migraine prevention in patients with documented failure to up to four migraine preventive medication classes (FOCUS): a randomised, doubleblind, placebo-controlled, phase 3b trial. Lancet Lond Engl 394:1030-1040

4. Mulleners W, Kim B, Láinez MJ et al (2019) A phase 3 placebo-controlled study of galcanezumab in patients with treatment-resistant migraine: results from the 3-month double-blind treatment phase of the conquer study. J Neurol Sci 405:128

5. Sacco S, Bendtsen L, Ashina M et al (2019) European headache federation guideline on the use of monoclonal antibodies acting on the calcitonin gene related peptide or its receptor for migraine prevention. J Headache Pain 20:6

6. Barbanti P, Aurilia C, Egeo G, Fofi L (2019) Erenumab: from scientific evidence to clinical practice-the first Italian real-life data. Neurol Sci Off J Ital Neurol Soc Ital Soc Clin Neurophysiol 40:177-179

7. Ornello R, Casalena A, Frattale I et al (2020) Real-life data on the efficacy and safety of erenumab in the Abruzzo region, Central Italy. J Headache Pain 21:32

8. Headache Classification Committee of the International Headache Society (1988) Classification and diagnostic criteria for headache disorders, cranial neuralgias and facial pain. Cephalalgia 8(Suppl 7):1-96

9. Goadsby PJ, Reuter U, Hallström Y et al (2017) A controlled trial of Erenumab for episodic migraine. N Engl J Med 377:2123-2132

10. Dodick DW, Ashina M, Brandes JL et al (2018) ARISE: a phase 3 randomized trial of erenumab for episodic migraine. Cephalalgia Int J Headache 38: 1026-1037

11. Tepper S, Ashina M, Reuter U et al (2017) Safety and efficacy of erenumab for preventive treatment of chronic migraine: a randomised, double-blind, placebo-controlled phase 2 trial. Lancet Neurol 16:425-434

12. Tepper SJ, Diener $\mathrm{H}-\mathrm{C}$, Ashina $\mathrm{M}$ et al (2019) Erenumab in chronic migraine with medication overuse: subgroup analysis of a randomized trial. Neurology. 92:e2309-e2320

13. Goadsby PJ, Holland PR (2019) An update: pathophysiology of migraine. Neurol Clin 37:651-671

14. Ornello R, Tiseo C, Frattale I et al (2019) The appropriate dosing of erenumab for migraine prevention after multiple preventive treatment failures: a critical appraisal. J Headache Pain [online serial] 20 Accessed at: https://www.ncbi.nlm.nih.gov/pmc/articles/PMC6822439/. Accessed December 26, 2019

\section{Publisher's Note}

Springer Nature remains neutral with regard to jurisdictional claims in published maps and institutional affiliations.

Ready to submit your research? Choose BMC and benefit from:

- fast, convenient online submission

- thorough peer review by experienced researchers in your field

- rapid publication on acceptance

- support for research data, including large and complex data types

- gold Open Access which fosters wider collaboration and increased citations

- maximum visibility for your research: over $100 \mathrm{M}$ website views per year

At BMC, research is always in progress.

Learn more biomedcentral.com/submissions 